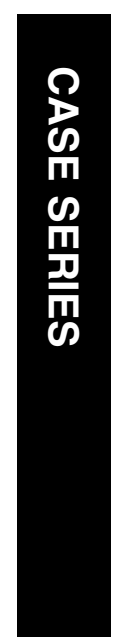

\title{
Unique phenotype in a Chinese family pedigree: ectopia lentis with varicose great saphenous vein
}

${ }^{1}$ Department of Ophthalmology, First Affiliated Hospital of Second Military Medical University, Shanghai, China

${ }^{2}$ Department of Ophthalmology, Peking Union Medical College Hospital, Beijing, China

${ }^{3}$ Department of Clinical Medicine, Second Military Medical University,

Shanghai, China

Correspondence: L Liu, Department of Ophthalmology, First Affiliated Hospital of Second Military Medical University,

168 Changhai Road,

Shanghai 200433,

P.R. China.

Tel: + 862181873558 ;

Fax: + 862165491531

E-mail: linliu@sh163.net

${ }^{4}$ These authors contributed equally to this work

Received: 31 January 2010 Accepted in revised form: 30 April 2010

Published online: 4 June 2010

\begin{abstract}
Objective To report a Chinese family affected with both ectopia lentis and varicose great saphenous vein.

Design Observational pedigree report. Participants The family with a total of 53 members in five generations. In the kindred there were 16 affected adults (including 6 deceased), of which 7 were male and 9 were female.

Main outcome measures Patients in this family showed an autosomal dominant trait of ectopia lentis and varicose great saphenous vein, occurring in four successive generations. The onset ages for lens dislocation were between 38 and 52 years. No cardiovascular abnormality was observed. Four patients underwent intracapsular lens extraction surgery.

Conclusions The phenotype of this family showed similarities with Marfan-related disorders. This is a unique phenotype of ectopia lentis with varicose great saphenous vein.

Eye (2010) 24, 1614-1617; doi:10.1038/eye.2010.82;

published online 4 June 2010
\end{abstract}

Keywords: ectopia lentis; varicose great saphenous vein; pedigree

\section{Introduction}

During the treatment for an adult patient with lens dislocation, we found the patient was also having varicose great saphenous vein (GSV). Further investigation discovered that there are more lens dislocation patients among five generations of this patient family who all have varicose GSV. It is a unique phenotype of ectopia lentis with varicose GSV in a Chinese family.

Case reports

Proband (III3; male, 52 years old). Sudden blurred vision appeared in his right eye on 31 August 2008. Eye examination showed visual acuity: right eye $5 / 20$, left eye $6 / 20$, and no improvement with correction. Slit-lamp microscope examination showed increased anterior chamber depth, Tyndall (-); iris tremor and lens opacity were observed. The right lens was subluxated downward and the upper edge could be seen after mydriasis (Figure 1a). The left lens was with mild dislocation toward the temporal side and the nasal side of lens edge could be seen after mydriasis (Figure 1b). There were moderate vitreous opacities, normal intraocular pressure, and no abnormalities in retina. The right eye axial length was $24.13 \mathrm{~mm}$. The patient also had varicose GSV (Figure 1c). Echocardiography was normal (Figure 1d). The patient was diagnosed as: subluxation (right), mild dislocation (left), and senile cataract. Later, the patient had the intracapsular lens extraction surgery on the right eye in our hospital.

The family has been living in Chongming county of Shanghai, China for generations.

All 53 family members in five generations belong to the Han ethnic group. There were 16 affected subjects, 7 men and 9 women; of which 6 are dead (causes of death were not due to cardiovascular diseases). There is no consanguinity marriage in this family. Intelligence evaluations of the family were normal.

From the outline of the pedigree (Figure 2), patients in this family pedigree have shown an autosomal dominant trait of lens dislocation and varicose GSV. The patients, found in four 

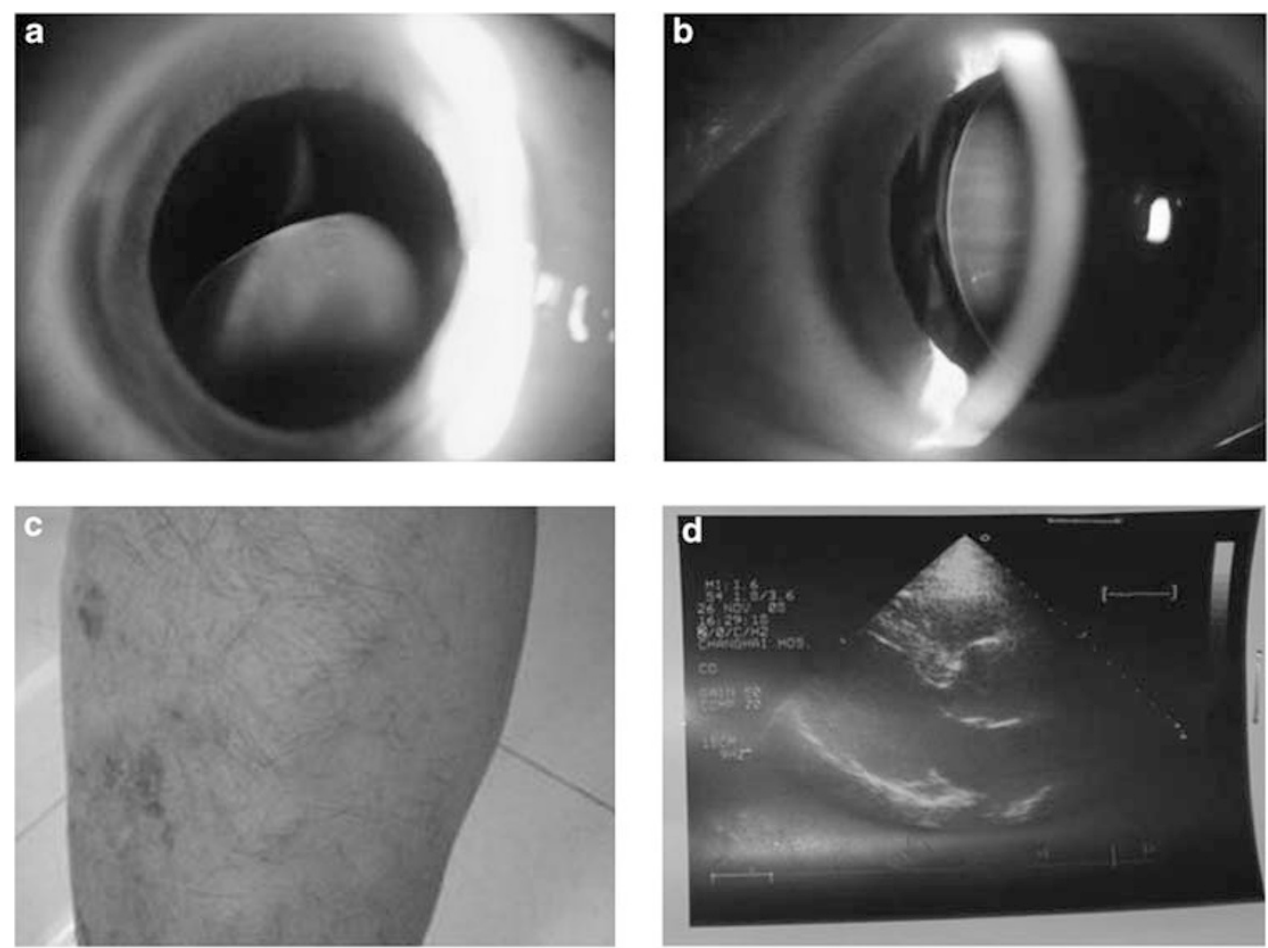

Figure 1 Patient III3 photos (a) right eye, (b) left eye, (c) varicose GSV, (d) echocardiograph (ascending aorta diameter: $2.1 \mathrm{~cm}$, aortic root diameter: $2.3 \mathrm{~cm}$. There was no abnormal finding in volumetric measurement of atrium or ventricle).

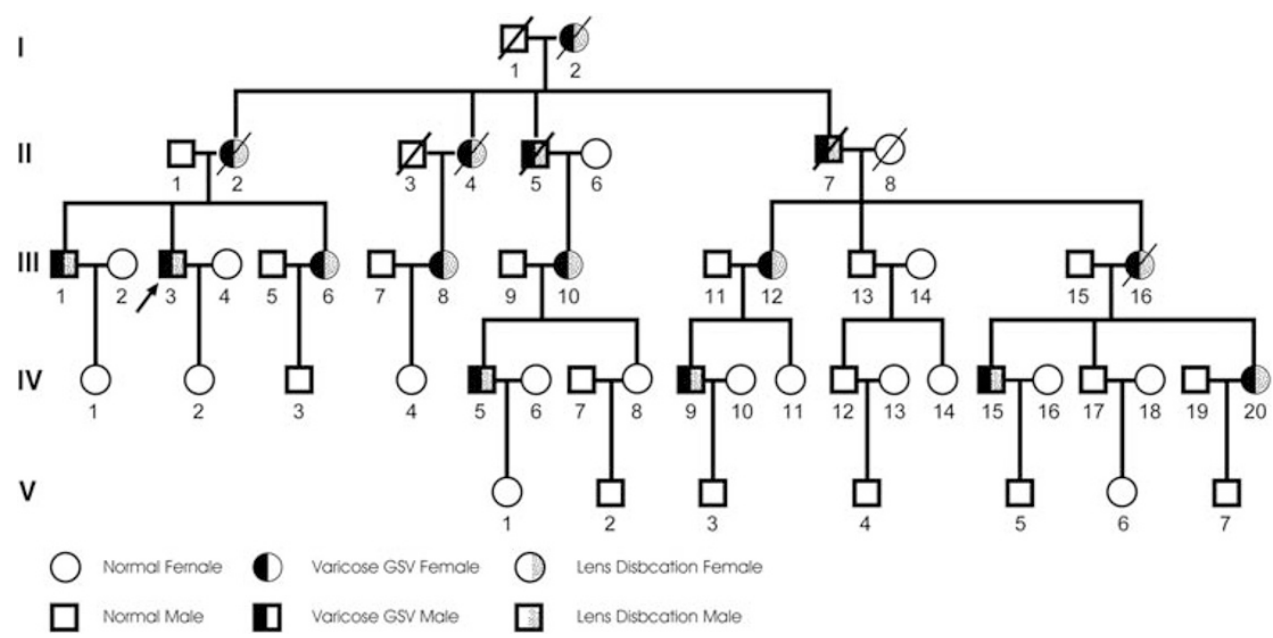

Figure 2 The pedigree (each family member can be identified by generation, labeled in roman numeral, and position in the generation, labeled in arabic numeral).

successive generations, with lens dislocation, all had varicose GSV (eg patient III3, Figure 1a-c; patient III12, Figure $3 \mathrm{a}$ and $\mathrm{b}$; patient III1, Figure $3 \mathrm{c}$ and $\mathrm{d}$ ), or vice versa. Blurred visions, without glaucoma and retinal detachment, were always the first symptom for lens dislocation patients. The lens dislocations were mostly to the temporal side at the beginning, then gradually down off to the vitreous and were always binocular with gradually increased opacities of lens cortexes over years. The onset interval for both eyes of each patient was usually within 1 year. The onset ages of lens dislocation were between 38 and 52 years, and those of varicose GSV were mostly around 40 years (only IIII2, Figure $3 a$ and b, was around 20 years). All patients showed no cardiovascular abnormality by echocardiograms. Within the family, phenotypic variation was also found (eg most 

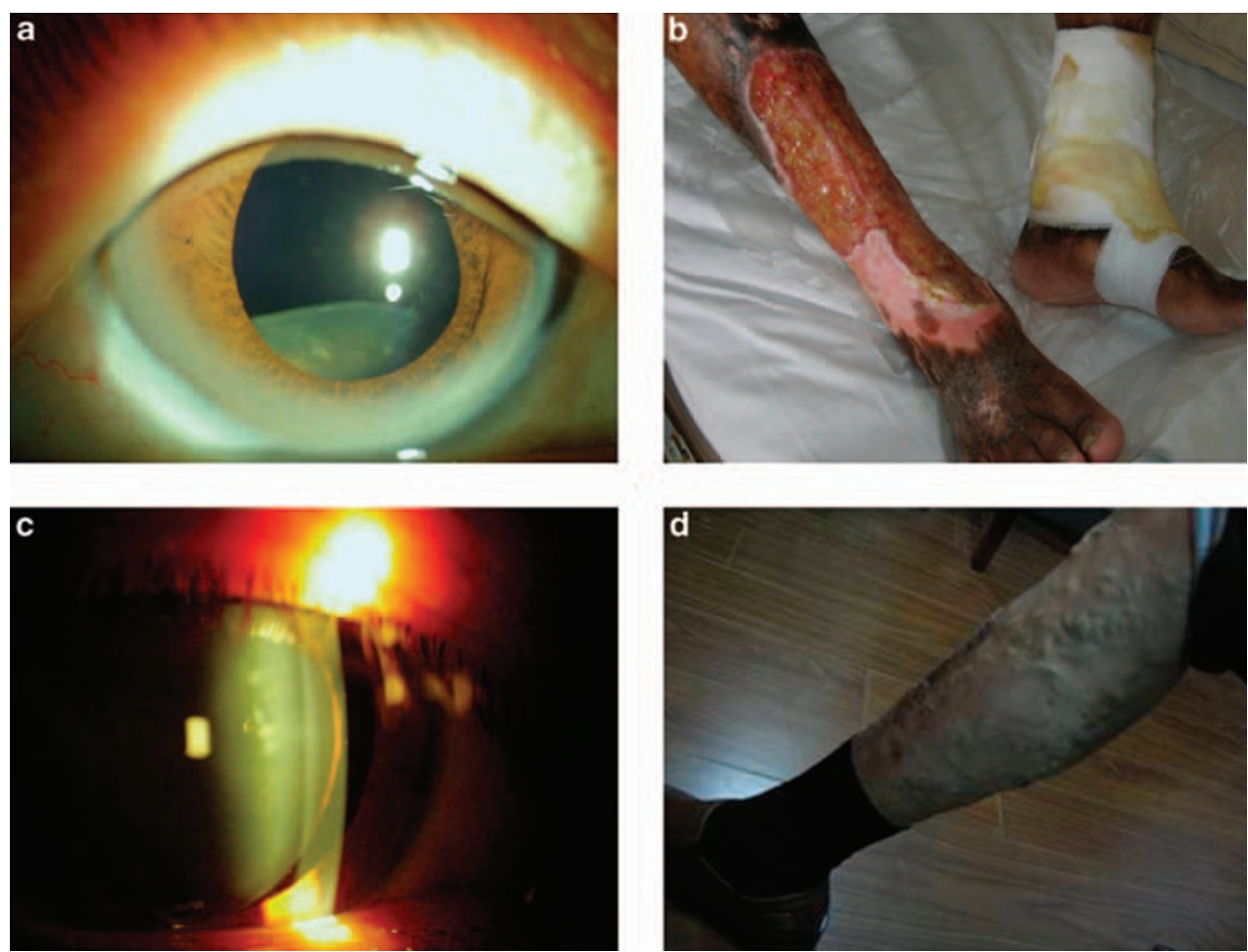

Figure 3 Patients photos (a) III12eye (b), III12 viricose GSV (III12 had varicose GSV surgery in 1986 and is now admitted to hospital for varicose GSV treatment), (c) III1 eye, (d) III1 varicose GSV (III1 is wearing stretch hose as varicose GSV treatment).

patients were myopia but two were emmetropia, and most were normal height but one was tall and with arachnodactyly).

Four patients have already had the intracapsular lens extraction surgery in our hospital. Another two patients with dislocated lens (IV9 and IV15) have also scheduled the surgery.

\section{Discussion}

Marfan syndrome (MFS) is a systemic disorder of connective tissue. Its manifestations involve eye, musculoskeletal, cardiovascular, and central nervous system. MFS and Marfan-related disorders show dominant inheritance and complete penetrance, but considerable clinical variation is seen both intra- and interfamilies. The main disease-causing gene is FBN1. ${ }^{1,2}$ MFS type 2 is caused by mutations in transforming growth factor- $\beta$ receptor types I and II (TGF $\beta$ R1 and TGF $\beta$ R2). ${ }^{3}$

Vascular disease in Marfan-related patients is characterized by elastic matrix abnormalities in the medial layer of the aortic wall, described as aortic aneurysm. It is interesting that Law $e t a l^{4}$ reported the disposition to aortic dilatation and dissection of a UK pedigree with varicose veins in male and female members in the teens, but no one had ocular lens dislocation. Our findings in this family provided a phenotype of ectopia lentis with varicose GSV without any aortic pathology found.

Ectopia lentis simplex is an autosomal dominant disease, which has also been reported relating to $F B N 1.5,6$ The lens dislocation is generally down to the nasal or temporal side. Ectopia lentis simplex is an eye-only disease that apparently differs from the manifestations of patients in this family.

FBN1 constitutes an important component of the extracellular microfibrils, which are found at the margins of maturing elastic fibres during embryogenesis. The cause of lens dislocation and varicose GSV in this family and whether FBN1 or TGF $\beta$ R2 is responsible for the phenotype are worth further study.

\section{Summary}

\section{What was known before}

- Aortic dilatation and dissection with varicose veins of a UK pedigree was reported. No report on ectopica lentis with varicose GSV.

What this study adds

- Unique phenotype from patients with lens dislocation and varicose GSV in a Chinese family pedigree.

\section{Conflict of interest}

The authors declare no conflict of interest. 


\section{References}

1 Ramirez F. Fibrillin mutations in Marfan syndrome and related phenotypes. Curr Opin Genet Dev 1996; 6: 309-315.

2 Ramirez F, Dietz HC. Marfan syndrome: from molecular pathogenesis to clinical treatment. Curr Opin Genet Dev 2007; 17: 252-258.

3 Singh KK, Rommel K, Mishra A, Karck M, Haverich A, Schmidtke J et al. TGFBR1 and TGFBR2 mutations in patients with features of Marfan syndrome and Loeys Dietz syndrome. Hum Mutat 2006; 27(8): 770-777.
4 Law C, Bunyan D, Castle B, Day L, Simpson I, Westwood G et al. Clinical features in a family with an $\mathrm{R} 460 \mathrm{H}$ mutation in transforming growth factor $\beta$ receptor2 gene. J Med Gent 2006; 43: 908-916.

5 Edwards MJ, Challinor CJ, Colley PW, Roberts J, Partington MW, Hollway GE. Clinical and linkage study of a large family with simple ectopia lentis linked to FBN1. Am J Med Genet 1994; 53: 65-71.

6 al-Salem M. Autosomal recessive ectopia lentis in tow Arab family pedigrees. Ophthalmic Paediatr Genet 1990; 11: 123-127. 